\title{
Quantitative predication on the water abundance of coal seam roof based on TEM
}

\author{
Kang Chen, Guoqiang Xue \\ Key Laboratory of Mineral Resources \\ Institute of Geology and Geophysics, CAS \\ Beijing, China \\ chenkang_igg@126.com
}

\begin{abstract}
With the depth of Coal mining deepen, it becomes a greater challenge to prevent and control water. Quantitative predication on the water abundance of coal seam rapid has become a serious problem. However, since inherent limitations of the method, only qualitative judgments can be done. Based on the numerical simulation, transient electromagnetic method and pumping tests are combined in this paper. Using many data of unit Inflow of drilling pumping test and apparent resistivity values measured near the borehole by TEM, the regression equation was set up. From the regression equation and the apparent resistivity values of remaining positions, quasi unit Inflows at other locations were quantitatively estimated, which can be used to quantitatively estimate the entire survey area. The result shows transient electromagnetic response can well reflect the changing nature of the aqueous layer of low resistivity. For complex aqueous region, finer quantitative information of the water abundance of aquifer can be given by quasi unit Inflow. The method in a coal mine in Inner Mongolia have been exploratory applications, and achieved rich water of coal seam roof of rapid quantitative assessment.
\end{abstract}

Key word-TEM; pumping test; water abundance; quantitative estimates

\section{INTRODUCTION}

Chinese coal mining is developing from the shallow to the deep zone at annual rate of $8-12 \mathrm{~m}$. With the current evaluation theory of water inrush coefficient, North China and East China region have entirely entered a stage that the mining of coal resources is threaten by water inrush damage $[1-3]$.

In this paper, we make the best of the advantage of sensitivity of the transient electromagnetic method to low resistivity body ${ }^{[4]}$ and combine it with pumping tests, then use regression analysis ${ }^{[5-6]}$ to establish the regression equation of water inflow of coal seam roof and apparent resistivity values, thereby based on the apparent resistivity obtained by transient electromagnetic method, we can determine unit water inflow $\hat{q}$ of outside the drilling region and make a comprehensive and detailed analysis of hydrogeological survey area so as to provide an important reference for the safety production of coal mining .

\section{FEASIBILITy ANALySES}

Using non-ground loop laid on the surface sends primary electromagnetic field of a certain-waveform emitted by pulse to the underground for central loop transient electromagnetic method. In intermittent period, observe the change of the secondary eddy current field over the time by a coil or grounding electrode receiver ${ }^{[7]}$. Analytic expression of the induced electromotive force at center position is ${ }^{[8]}$ :

$$
V(t)=-2 \pi \mu a^{2} I \int_{0}^{\infty} L_{p}\left(\frac{1}{\lambda+u}\right) \lambda \mathrm{J}_{1}(\lambda a) \mathrm{J}_{1}(\lambda b) \mathrm{d} \lambda
$$

in the formula,

$$
\frac{1}{\lambda+u}=\frac{\lambda}{\mu_{0} \sigma s}-\frac{\sqrt{\left(s+\lambda^{2} / \mu_{0} \sigma\right) / \mu_{0} \sigma}}{s}
$$

The earth permeability is taken as the vacuum permeability $\mu_{0}, I$ is emission current, $L_{p}$ is the Laplace transform operator, $\sigma$ is the earth conductivity, $\lambda$ is the integral variable, $J_{1}$ is the first order Bessel function, $\mathrm{a}$ and $\mathrm{b}$ represent the radius of the transmission line and the receiving loop, respectively, for the rectangular wire frame, there is $a=\sqrt{\mathrm{A} / \pi}$.

In order to study the influence of the change of aquifer properties on the transient electromagnetic response characteristics, we designed three theoretical models (TABLE I) according to the similarity criterion.

TABLE I THEORETICAL MODEL PARAMETER TABLE

\begin{tabular}{cccccc}
\hline & $h_{1}(\mathbf{m})$ & $\rho_{1}(\Omega \mathrm{m})$ & $h_{2}(\mathbf{m})$ & $\rho_{2}(\Omega \cdot \mathrm{m})$ & $\rho_{3}(\Omega \cdot \mathrm{m})$ \\
\hline Model A & 100 & 100 & 30 & 10 & 200 \\
\hline Model B & 100 & 100 & 30 & 20 & 200 \\
\hline Model C & 100 & 100 & 10 & 20 & 200 \\
\hline
\end{tabular}

According to the formula above, we calculated the transient electromagnetic response of model A, model B and model $\mathrm{C}$, and shown as Fig.1 in the double logarithmic coordinates. By Fig.1a, we can see that the value of the induced electromotive force measured on surface has an obvious change: compared with model $\mathrm{B}$, the curve of model A decreases in the middle and rises again with the depth increasing. The slope of the curve also changes and there is a clear reflection on the middle decreasing low resistivity layer. 
Similarly, we can see from Fig.1b, compared with model B, curve of model $\mathrm{C}$ has an obvious uplift in the middle which can also reflect thickness decreasing of the middle low resistivity layer. Thus, we can conclude that when the property of the aquifer changes, then the surface observation data will reflect the corresponding changes.
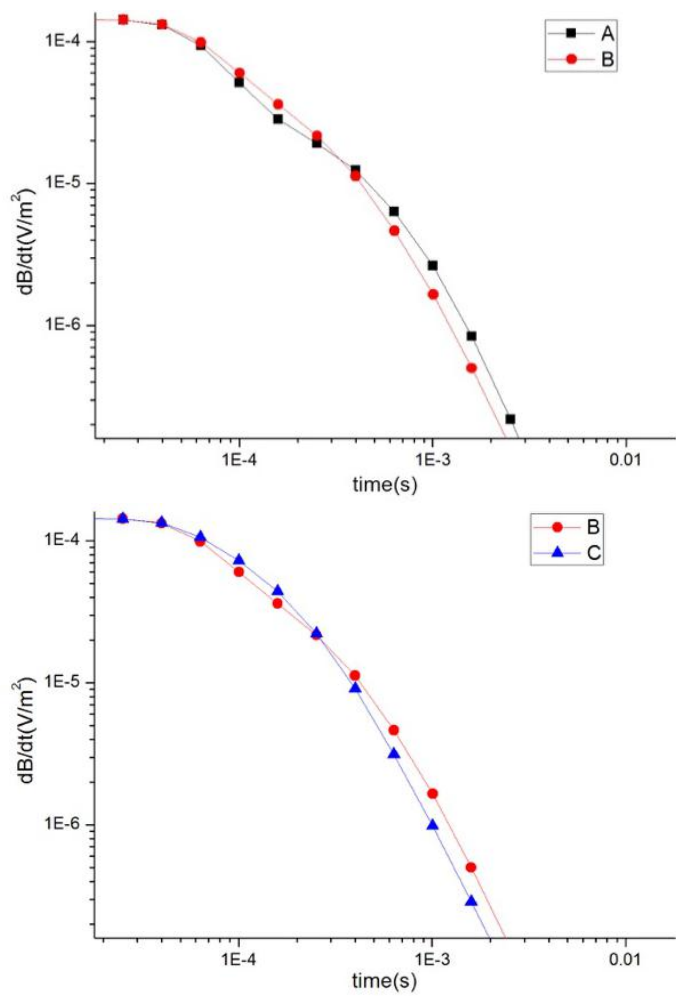

Fig.1 the comparison diagram of modes of transient electromagnetic response

Similarly, by pumping test we can directly obtain the quantitative information of underground water yield property. It is used to quantitatively determine water yield property of aquifer in accordance with the unit inflow.

For the target to be measured, we carry out pumping test for three times with aquifer drawdown and get data of flux $Q$ and drawdown $S$. Using the least square method or the graph method to obtain the relationship curve $Q=f(S)$, then according to the curve $Q-S$ to determine the water inflow of pumping hole for aquifer drawdown of $10 \mathrm{~m}$, use the formula (2) to calculate water inflow of hole diameter of $91 \mathrm{~mm}$ and divide it by 10 to obtain unit water inflow.

$$
Q_{91}=Q_{孔}\left(\frac{\lg R_{孔}-\lg r_{孔}}{\lg R_{91}-\lg r_{91}}\right)
$$

In the formula, $Q_{91} 、 R_{91} 、 r_{91}$ represent water inflow of radius $91 \mathrm{~mm}$ borehole, influencing radius and the drilling hole radius, respectively. $Q_{孔} 、 R_{孔} 、 r_{孔}$ denote the water inflow of radius $r$ borehole, the influence radius and the drilling hole radius. In the 'Prevention and Control Regulations of Coal Mine water', the unit inflow is a kind of classification standard of mine hydrogeological types, and in the actual production process, it is also used to quantitatively determine water yield property of aquifer in accordance with the unit inflow.

From the above analysis, we can know that transient electromagnetic response and unit inflow can reflect the change of aquifer properties, and determine the relationship of the two variables, so we can find a fast and accurate assessment method.

\section{The Fast and Quantitative Estimation Method}

By using the method of regression analysis, we combine the apparent resistivity obtained by transient electromagnetic method with the unit water inflow obtained by pumping test to establish the statistic model, and use it to study the statistic relationship between them in the survey area. The characteristic of measured data has been analyzed and select the appropriate model to establish regression equation.

$$
q=f\left(\rho_{i}, \theta\right)+\varepsilon_{i}
$$

regression analysis of the general equation is described as:

$$
y=\varphi\left(x_{1}, x_{2}, \ldots, x_{m}, \beta_{1}, \beta_{2}, \ldots, \beta_{i}\right)+\varepsilon
$$

for a given set of observations $\left(x_{i}, y_{i}\right), i=1,2, \ldots, n$, we can rewrite the formula

$$
y_{i}=f\left(x_{i}, \theta\right)+\varepsilon_{i} i=1,2, \ldots, n
$$

Among them, $y_{i}$ is the dependent variable, namely the unit inflow $\mathrm{q} ; \mathrm{X}_{\mathrm{i}}$ is the independent variable, namely the apparent resistivity $\rho ; \theta$ is for the vector of unknown parameters; $\varepsilon_{i}$ is random error item meeting the independent identical distribution assumption. In case of assumption that the function $\mathrm{f}$ is continuously differentiable to the parameters $\theta$, use differential method to establish the normal equation group, in order to seeking the least $\hat{\theta}$ of the following equation

$$
Q(\theta)=\sum_{i=1}^{n}\left(y_{i}-f\left(x_{i}, \theta\right)\right)^{2}
$$

Derive partial derivation of the function $\mathrm{Q}$ to the parameters $\theta_{\mathrm{j}}$, and let it be 0 , get $p+1$ equations

$$
\left.\frac{\partial Q}{\partial \theta_{j}}\right|_{\theta_{j}=\hat{\theta}_{j}}=-\left.2 \sum_{i=1}^{n}\left(y_{i}-f\left(X_{i}, \hat{\theta}\right)\right) \frac{\partial f}{\partial \theta_{j}}\right|_{\theta_{j}=\hat{\theta}_{j}}=0 \quad j=0,1,2, \ldots, p
$$

The least squares estimation $\hat{\theta}$ is the solution of formula, and finally we will obtain the regression equation of $\rho$ and $\mathrm{q}$.

Finally, by using the transient electromagnetic method to determine the area to be evaluated, according to the known coal seam roof of aquifer to determine calibration time $t$ and calibration apparent resistivity $\rho$ of the roof aquifer in each measuring point, then substitute these values to obtain the regression equation, and through this method, we can get unit water inflow $\hat{\mathrm{q}}$ of each point of coal seam roof of the area to be estimated (flow chart below). 


\section{APPLICATION EXAMPLES}

The formation of survey area is relatively flat and angle is less than 5 degrees. The main coal seam is located in the upper part of Klym group, stable and continuous development, the maximum thickness $9.41 \mathrm{~m}$, minimum thickness $0.18 \mathrm{~m}$, the average thickness $5.46 \mathrm{~m}$, the upper part has a very thick gravel aquifer which is connected with surface river, and the aquifer is thin, the whole region's development is not stable the and form complex hydrogeological conditions

In order to achieve accurate quantitative estimates of underground water yield property, in this test, arranged for hydrological 11 boreholes and did pumping test to obtain the unit water inflow data. At the same time, we applied V8 multifunctional electromagnetic instrument of Phoenix company of Canada's, arranged 17 survey lines along the north-south direction, the exploration grid is for $40 \mathrm{~m}$ (line spacing) $\times 20 \mathrm{~m}$ (spot spacing).

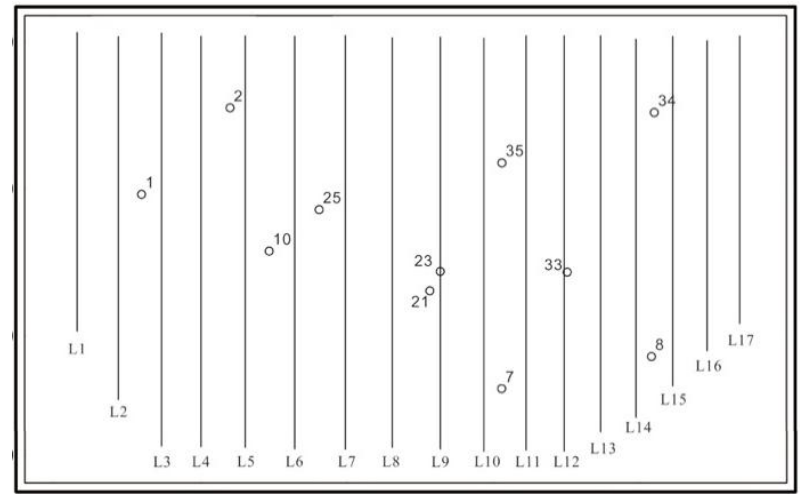

Fig.2 engineering layout

According to the analysis of the hydrological-geophysics model in the region, we select $170 \mathrm{~m}$ for detection depth. Using the above regression analysis method to make scatter plot of the apparent resistivity and the unit inflow (Fig. 3):

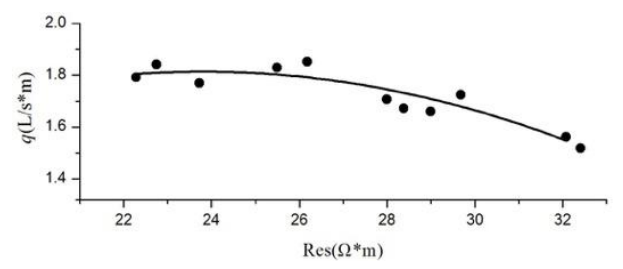

Fig.3 regression model

From the scatter plot, we can see that sample data roughly fall in near a parabola, indicating that there is an obvious correlation between water inflow and apparent resistivity. But the relationship did not reach the degree for that a given resistivity will be exactly corresponding to a

\section{CONCLUSIONS AND OUTLOOK}

The research results show that by establishing the relation between apparent resistivity and unit water inflow to obtain the quasi unit water inflow, and it is effective to solve unique unit inflow. So, by solving the regression equations, we can get regression model between two variables:

$$
y=-0.003 x^{2}+0.185 x-0.4
$$

After processing the whole region's transient electromagnetic data to obtain the apparent resistivity values of the corresponding depth, use the method of establishing regression equation finally to get the quasi unit water inflow $\hat{q}$ at the corresponding position. To better illustrate the feasibility and superiority of the method, we made $\hat{q}$ contour map and put it together with the contour map of the actual measured unit water inflow and did a comparison, as shown in Fig.4.

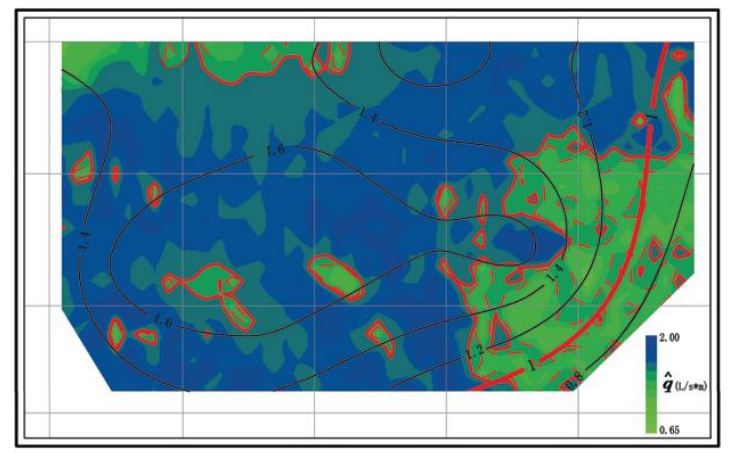

Fig.4 the contour comparison

In figure, the color area represents the unit water inflow strength, the values become bigger from green to blue value, and we mark the weak water-bearing area with a red line. Numerical lines represent the contour of measured unit water inflow $q$, according to the hydrogeology to delineate the standard of water-rich area, the area of units water inflow more than $1 \mathrm{~L} / \mathrm{s} \cdot \mathrm{m}$ is for strong water-rich district, the thick red lines in figure represent the unit inflow value for $1 \mathrm{~L} / \mathrm{s}^{\cdot} \mathrm{m}$, it is not difficult to find:

- two methods show a broadly consistent reflection on the strength of water yield property of aquifer: in the central, west and northeast zone of the survey area, water inflow of aquifer is bigger, having a great influence on the mining of coal seam; In the eastern and southeast part of the survey area, the water yield property of aquifer is weak.

- the data of actual unit water inflow is less due to the influence by cost, in contour map and most region data is for data interpolation, not real data. Quasi unit water inflow has a better reflection in detail. By Fig.6, we can see that there are two obvious low water inflow zones in the center of the survey area, indicating that the underground aquifers are not uniform, which is in conformity with the reality.

the problem of the less of measured unit inflow data and inaccurate judgment of the region, and it solves not only the problem that the electromagnetic method can now only give a qualitative judgment of water-rich intensity but also improves the efficiency, saves the cost, and can truly reflect the characteristics of aquifers. 


\section{REFERENCES}

[1] Peng Suping. 2008. Present study and development trend of the deepen coal resource distribution and mining geologic evaluation: Coal, 17(2), 1-11.

[2] Wu Qiang, Cui Fangpeng, Zhao Suqi, et al. 2013. Type classification and main characteristics of mine water

[3] Hu Weiyue, Tian Gan. 2010. Mine water disaster type and prevention and control counter measures in China: Coal Science and Technology, 38(1), 92-96

[4] Christensen, N.B., Sørensen, K.I., 1998. Surface and borehole electric and electromagnetic methods for hydrogeological investigation: European Journal of Environmental and Engineering Geophysics, 3, 75-90.
[5] K. Vasanth Kumar, K. Porkodi , F. Rocha. Isotherms and thermodynamics by linear and non-linear regression analysis for the sorption of methylene blue onto activated carbon: Comparison of various error functions: Journal of Hazardous Materials, 2008, 794-804.

[6] Xu Qun.2009. The research on non-linear regression analysis methods: M.S. thesis, Hefei: Hefei University of Technology.

[7] Nabighian, M.N. 1991.Electromagnetic methods in applied geophysics. Vol.2: Application. Tulsa, Oklahoma: Society Exploration Geophysical.

[8] Shi Xianxin. 2005. Research on shielding of low-resistivity layer in prospecting by transient eletromagnetic method: D.S. thesis, Xi'an: China Coal Research Institute 